\title{
A Flow Scheduler Architecture
}

\author{
Dinil Mon Divakaran ${ }^{1, \star}$, Giovanna Carofiglio ${ }^{2}$, Eitan Altman $^{3, \star \star}$, \\ and Pascale Vicat-Blanc Primet ${ }^{1}$ \\ 1 INRIA / Université de Lyon / ENS Lyon, \\ LIP, ENS Lyon, Lyon - 69007, France \\ \{Dinil.Mon.Divakaran, Pascale.Primet\}@ens-lyon.fr \\ 2 Alcatel-Lucent Bell Labs \\ Giovanna.Carofiglio@alcatel-lucent.com \\ 3 INRIA \\ Eitan.Altman@sophia.inria.fr
}

\begin{abstract}
Scheduling flows in the Internet has sprouted much interest in the research community leading to the development of many queueing models, capitalizing on the heavy-tail property of flow size distribution. Theoretical studies have shown that 'size-based' schedulers improve the delay of small flows without almost no performance degradation to large flows. On the practical side, the issues in taking such schedulers to implementation have hardly been studied. This work looks into practical aspects of making size-based scheduling feasible in future Internet. In this context, we propose a flow scheduler architecture comprising three modules - Size-based scheduling, Threshold-based sampling and Knockout buffer policy - for improving the performance of flows in the Internet. Unlike earlier works, we analyze the performance using five different performance metrics, and through extensive simulations show the goodness of this architecture.
\end{abstract}

Keywords: Scheduling, Sampling, QoS, Future Internet, Architecture.

\section{Introduction}

Recent works have advocated the importance of networks being 'flow-aware'. Bonald et al. have listed the need for having a flow-aware architecture [1]. In a flow-aware network, the performance is measured at flow level. This is in line with the utility of end-users, where e.g., the delay of small flows, throughput of large flows, instantaneous rate of streaming traffic etc. are most often more important than packet-level QoS metrics. In this context, our goal is to come up with a flow scheduler architecture for improving the delay performance of small (and middle size) flows. The current Internet architecture has a FCFS scheduler and Droptail buffer at each of its nodes. These, along with the fact that most

\footnotetext{
* Corresponding author.

** This work was done in the framework of the INRIA and Alcatel-Lucent Bell Labs Joint Research Lab on Self Organized Networks.
} 
of the flows in the Internet are carried by TCP, makes this current architecture biased against small TCP flows for the following reasons. (i) A packet loss to a small flow most often results in a timeout due to the small 'congestion window' (cwnd) size; whereas, a large flow is most probably in the congestion avoidance phase, and hence has large cwnd size. Therefore, for a large flow, packet losses are usually detected using duplicate ACKs, instead of timeouts, thus avoiding slow-start. (ii) The increase in round trip time (RTT) due to large queueing delays hurts the small flows more than the large flows. Again, for the large flows, the large cwnd makes up for the increase in RTT; whereas, this is not the case for small flows.

These problems being well-known, researchers have explored scheduling algorithms that give priority to small flows. They range from SRPT (Shortest Remaining Processing Time) 2] to LAS (Least Attained Service) 3] to MLPS (Multi-level Processor Sharing) scheduling policies [4. While scheduling algorithms give priority in time, buffer management policies give priority in space. Guo et al. showed the gain in performance attained by giving space priority to small flows [5]; but it is a stand-alone concept that does not consider giving timepriority to small flows. We argue that it is important to give priority in both time and space to small flows, in order to reduce the delay as well as timeouts faced. To the best of our knowledge, LAS scheduling policy is the only policy that gives space priority to packets of small flows [6], thereby giving priority in both time and space. It does so by inserting incoming packet in the appropriate position and dropping from the tail whenever the buffer is full. But, it has been observed that LAS is unfair to very large flows [7]. Moreover, it is challenging to perform a strict ordering of packets of each flow at high line rates.

This work proposes a flow scheduler architecture, that gives priority in time as well as space to small flows, and uses sampling for performing size-based scheduling. To be precise, our flow scheduling architecture combines three essential modules that help in improving the delay performance of flows:

1. Generalized size-based scheduling;

2. Threshold-based sampling;

3. Knockout buffer policy.

The motivation for such an architecture is given in Section 2, where we also summarize related works. The architecture is detailed in Section 3. We perform extensive simulations and compare different performance metrics to show how each of these three strategies contributes in improving the performance of small flows, without affecting the performance of large flows. Unlike most previous works, where the performance was analyzed using just one metric (usually the conditional mean response time), we consider five different metrics. They are:

1. Conditional mean completion time of small flows;

2. Number of timeouts encountered by small flows;

3. Mean completion time for range of flow sizes;

4. Mean completion time for small flows, large flows and all flows;

5. Maximum completion time of small flows. 
The goal of simulations and the setting are described in Section 4. The benefits of using the Knockout policy are analyzed in Section 5] In Section 6, we evaluate the proposed flow scheduler architecture and compare with other schemes.

\section{Related Works and Motivation}

The literature in this research area being vast, we limit the references to a small but important subset. A large number of researchers have considered giving priority in time to small flows. These have given rise to the study of scheduling disciplines like SRPT, LAS and MLPS disciplines in the context of Internet flows. While SRPT requires the knowledge of flow size in advance [2, LAS is a 'blind' scheduling policy — requires no in-advance information on flow size [3]. These differentiating policies perform better in terms of delay, when compared to the naive PS (processor sharing) system 1 . The MLPS scheduling discipline is a generalized version with high flexibility, having $N$ different priority levels distinguished by $N-1$ thresholds, and strict priority among these levels 789 .

The drawbacks of LAS policy, such as unfairness and scalability issue, have motivated researchers to explore other means of giving priority to small flows, one such being the strict $P S+P S$ model proposed in [7]. The $P S+P S$ model, as the name indicates, uses two $P S$ queues, with priority between them. The first $\theta$ packets of every flow are served in the higher priority queue $\left(Q_{1}\right)$, and the remaining in the lower priority queue $\left(Q_{2}\right)$. The service discipline is such that, $Q_{2}$ is served only when $Q_{1}$ is empty. Therefore, it is a strict $P S+P S$ model. This work also takes a step forward in performance analysis of size-based scheduling systems, by analyzing another metric — maximum response time — other than the usual conditional mean response time. In addition, the authors proposed an implementation of this model; but it relies on TCP sequence numbers, requiring them to start from a set of possible initial numbers. This not only makes the scheme TCP-dependent, but also reduces the randomness of initial sequence numbers. Again, this is another work which does not account for space priority for small flows.

Authors of [5] considered prioritizing small flows in space. This is achieved by preferentially treating small flows inside the bottleneck queue which implement RIO (RED with In and Out). Small and large flows were assigned different drop functions. To facilitate this, they proposed an architecture where the edge routers mark packets as belonging to small or large flow, using a threshold-based classification. With priority given only in space, the performance gains in terms of average response times (apart from analyzing the fairness) is not complete.

We observe that most of the works dealing with giving preferential treatment based on size (or age) assume that the router keeps per-flow information. In fact, this assumption is challenged by the scalability factor, as the number of flows in progress is in the order of hundreds of thousands under a high load. One solution is to use sampling to detect large flows (thus classifying them), and

\footnotetext{
${ }^{1}$ At the flow level, the queues in the Internet are generally modelled as an $M / G / 1-P S$
} system, even when the queue is served using a FCFS policy at the packet level. 
use this information to perform size-based scheduling. Since the requirement here is only to differentiate between small and large flows, the sampling strategy need not necessarily track the exact flow size. A simple way to achieve this is to probabilistically sample every arriving packet, and store the information of sampled flows along with the sampled packets of each flow 10. SIFT, proposed in 11, uses such a sampling scheme along with the $P S+P S$ scheduler. A flow is 'small' as long as it is not sampled. All such undetected flows go to the higher priority queue until they are sampled. The authors analyzed the system using the 'average delay' (average of the delay of all small flows, and all large flows) for varying load, as a performance metric. Though it is an important metric, it does not reveal the worst-case behaviour in the presence of sampling. This is more important here, as the sampling strategy can have false positives; small flows if sampled will be sent to the lower priority queue. In such scenarios, it is necessary to compare other performance metrics, which we listed earlier.

\section{Architecture}

This section describes the modules of the architecture, and the cost of implementing this architecture.

\subsection{The Modules}

The flow scheduler architecture consists of three functional modules: a size-based scheduler, a threshold-based sampling technique to detect large flows, and a Knockout buffer policy giving space priority to small flows.

Size-Based scheduling: Since sampling introduces errors in the detection of large flows, thereby permitting misclassification, using a strict priority-scheduling strategy is not advisable. Therefore, we take a generalized model of the strict $P S+P S$ scheduling, called generalized size-based scheduling, or simply SB scheduling. As before, packets of all flows are served in $Q_{1}$, as long as the ongoing size is less than $\theta$ packets. Once the ongoing flow size crosses $\theta$, it is queued in $Q_{2}$. But instead of giving the whole capacity to $Q_{1}$, only a fraction of the capacity is assigned to $Q_{1}$. That is, the high priority queue is assigned a weight $0 \leq w \leq 1$. If $C$ is the link capacity, $Q_{1}$ and $Q_{2}$ are serviced at rates $w C$ and $(1-w) C$ respectively, whenever the queues are not empty. If $Q_{1}$ is empty, $Q_{2}$ is served at full capacity $C$. We assume that the scenario of $Q_{2}$ being empty and $Q_{1}$ being non-empty is a rare possibility. Note that, if $w=1$, this becomes the $P S+P S$ scheduling policy. The scheduling module in the figure is shown as deciding which queue to dequeue based on the parameter $w$.

Threshold-Based sampling: For the sampling part, we use the well-studied 'Sample and hold' strategy proposed for detecting large flows [12]. It works as follows. For every sampled packet, a flow entry is created in the flow table if it does not exist. A packet of $s$ bytes is sampled with a probability $p$, which is 
expressed in terms of byte-sampling probability $\beta$. We have, $p=1-(1-\beta)^{s}$. When a packet arrives, a flow table lookup is performed. If the arriving packet is found to be part of an existing flow, the flow-size counter in the flow table is updated. Thus, for each sampled flow, there is a counter that maintains the estimated size. This process is performed during every measurement interval. Thresholds are used to reduce false positives, and to preserve continuing large flows across intervals. Observe that the flow-table lookup is done for every arriving packet, and size update is performed for every detected flow. This is costly in terms of processing, but reduces the flow table's size considerably (to a few thousands of entries). Therefore, it is possible to use SRAM to store the flow table for efficient lookups. A useful property of this sampling strategy is that, since the estimated size is never greater than the actual flow size, by choosing an appropriate threshold, false positives can be completely avoided.

Knockout buffer policy: The third part is the Knockout buffer policy for giving space priority to small flows. Though there is only one single physical queue, it is shared by two virtual queues, one for enqueueing packets of flows classified as small, the other for enqueueing packets of flows classified as large. These correspond to the two queues $Q_{1}$ and $Q_{2}$ described earlier. The policy is different from Droptail only during packet discard instants [13. Upon the arrival of a packet when the physical buffer is full, the Knockout policy operates thus: if the packet is for $Q_{2}$ (i.e., the system has classified it as belonging to a large flow), it is dropped. If the arriving packet is for $Q_{1}$ (i.e., the system has classified it as belonging to a small flow), the last packet from $Q_{2}$ is 'knocked out' making space for this new packet. In the scenario of $Q_{2}$ being empty (i.e., the physical buffer has packets of only flows classified as small), the arriving packet is dropped. Assuming most large flows are carried by TCP, dropping a packet from a large flow is meaningful as it will be retransmitted by the TCP source.

Fig. 1 gives a pictorial representation of the architecture. An arriving packet first goes to the sampling module, which does a flow-table lookup. Packet sampling and flow-table update are performed if necessary. The queueing module decides to queue the packet in $Q_{1}$ or $Q_{2}$ based on the flow-size estimate

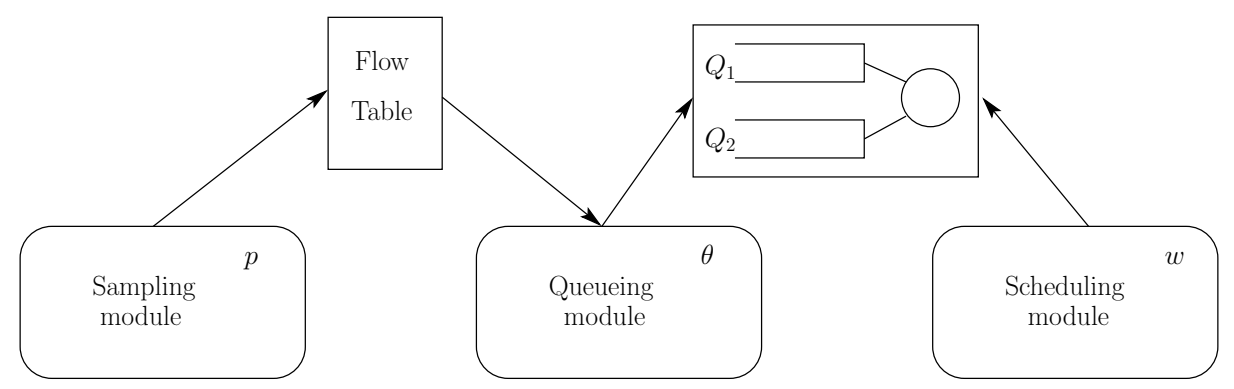

Fig. 1. Flow scheduler architecture 
available from the flow table and the parameter $\theta$. If the physical buffer is full, the Knockout policy is used to select the packet to be dropped. The scheduling module uses the weight parameter $w$ to perform SB scheduling.

\subsection{Implementation Cost}

SB scheduling requires two queues. These can be implemented as virtual queues, on top of the physical queue. The scheduling of packets as such can then be implemented by assigning weights to these queues.

For the sampling, an SRAM with sufficient size to hold the flow table is required. There is extra processing for updating the flow size of detected flows. The flow-table lookup can be combined along with route-table lookup.

Knockout policy uses the two virtual queues, $Q_{1}$ and $Q_{2}$, with $Q_{1}$ being the higher priority queue. Observe that a virtual queue can grow to the actual size of the physical queue with the other virtual queue being empty. To be able to knock out an already queued packet from $Q_{2}$, the tail of $Q_{2}$ needs to be tracked. All these can be achieved if the physical queue is implemented as a linked list, and pointers to the head and tail of the two virtual queues are maintained. This deviates from the simplest way of implementing a queue as a circular buffer, thus adding extra overhead in maintaining the linked list.

\section{Simulation}

\subsection{Goal}

The goal of the simulations is to evaluate the performance of the flow scheduler architecture. As described earlier, small flows are biased against large flows when it comes to timeouts. Hence, we are interested in analyzing not only the improvement in delay performance, but also the reduction in number of timeouts faced by small flows. On the other hand, since prioritizing small flows should not adversely affect large flows, the mean completion times of large flows conditioned on their flow sizes are also analyzed. To see the improvement over today's Internet architecture, we compare results with the FCFS scheduler. Along with the FCFS scheduler, the buffer policy used in all simulations here is Droptail (as is the case in Internet nodes), though not stated explicitly in figures.

\subsection{Settings}

Simulations are performed using NS-2. A dumbbell topology, representing a single bottleneck link connecting source-destination pairs, was used throughout. The bottleneck link capacity was set to $1 \mathrm{Gbps}$, and the capacities of the source nodes were all set to $100 \mathrm{Mbps}$. The delays on the links were set such that the base RTT (consisting of only propagation delays) is equal to $100 \mathrm{~ms}$. The size of the bottleneck queue is set in bytes, as the bandwidth delay product (BDP) for $100 \mathrm{~ms}$ base RTT. There were 100 node pairs, with the source nodes generating 
flows according to a Poisson traffic. The flow arrival rate is adapted to have a packet loss rate of around $1.25 \%$ with FCFS scheduler and Droptail buffer. Flow sizes are taken from a Pareto distribution with shape $\alpha=1.1$, and mean flow size set to $500 \mathrm{~KB}$.

All flows are carried by TCP, in particular, using the SACK version. Packet size is kept constant and is equal to $1000 \mathrm{~B}$. For simplicity, we keep the threshold in packets; $\theta$ is set to 25 packets in all the scenarios, unless explicitly stated otherwise. For post-simulation analysis, we define 'small flow' as a flow with size less than or equal to $20 \mathrm{~KB}$, and 'large flow' as one with size greater than $20 \mathrm{~KB}$. Here the flow size is the size of data generated by the application, not including any header or TCP/IP information. Also note that, a small flow of $20 \mathrm{~KB}$ can take more than 25 packets to transfer the data, as it includes control packets (like $S Y N, F I N$ etc.) and retransmitted data packets.

\section{Performance Analysis of the Scheduler Using Knockout}

Here we analyze SB scheduler using Knockout buffer policy, but without sampling. In this case, flows are accurately classified as small and large by tracking the ongoing size of the flow. The focus of this section is to show the importance of having the Knockout buffer policy.

Before choosing the weights, we present an observation. First, it should be noted that, by giving priority to small flows, a policy essentially tries to keep the corresponding buffer for small flows almost empty. With this in mind, we conducted simulations to analyze the average occupancy of $Q_{1}$ for different weights. The result is shown in Fig. 2, The number of packets of small flows in queue is almost constant for weights $w \geq 0.6$. Hence, any $w \geq 0.6$ should give close performance for small flows. Dynamically adapting $w$ according to the buffer occupancy being outside the scope of this work, we set $w$ to 0.8 for SB scheduler in our simulations. The other scenario considered is with $w$ set to 1.0 ; thus we also analyze the strict $P S+P S$ system. Even when there is no sampling involved, we see that there is no notable gain in using a strict SB scheduler.

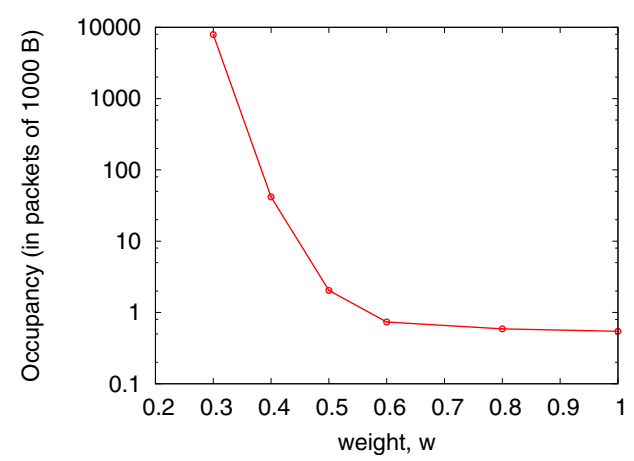

Fig. 2. Average occupancy for $Q_{1}$ for different weights 


\section{$5.1 \quad$ Results}

Fig. 3(a) shows the mean completion time conditioned on the flow sizes, for small flows. The naive packet-level FCFS scheduling policy is shown as a comparison. The other curves correspond to SB scheduling with different weights, and with and without the Knockout policy. A value of ' 0 ' for $K O$ implies that the Knockout policy is not in use, and ' 1 ' implies the contrary. The figure shows the goodness of size-based scheduling compared to the FCFS scheduling. Knockout buffer policy is seen to complement the SB schedulers. Observe also that, with this metric, there is no notable difference using a weight of 0.8 or 1.0 .

Fig. 3(b) indicates that the large flows are not affected by giving priority to small flows (both in space and time). In fact, it can be seen in Fig. 4(a) that the SB scheduler with $w=0.8$ and $K O=1$, gives the same mean delay for very large flows, as does the FCFS scheduler. Fig. 4(a) plots the mean completion time of flows within different size ranges (e.g., 0-20 packets, 21-200 packets etc.). The mean values show that, in general, the SB scheduler also performs better for medium flows (those with a size around 2000 packets).

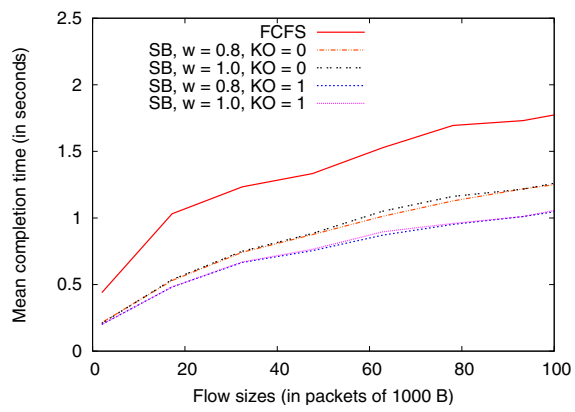

(a) small flows

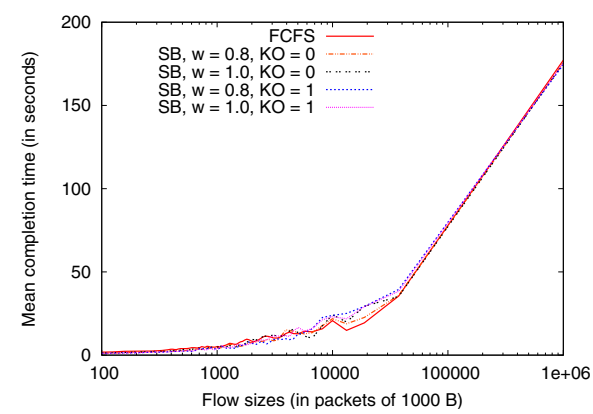

(b) large flows

Fig. 3. Conditional mean completion time

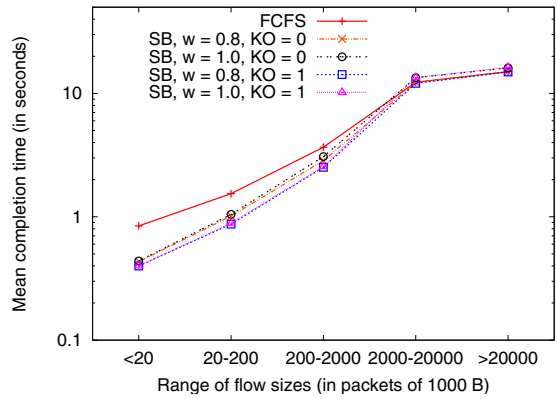

(a) Mean completion time

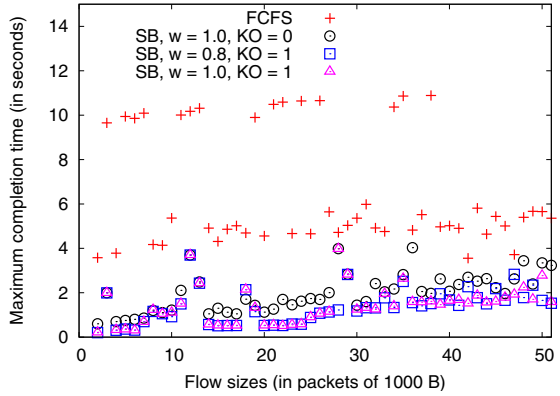

(b) Maximum completion time

Fig. 4. Other metrics 
Table 1. Comparison of different metrics

\begin{tabular}{|c||c|c|c|c|}
\hline Metrics & $\begin{array}{c}\text { small } \\
\text { TOs }\end{array}$ & $\begin{array}{c}\text { small } \\
C T\end{array}$ & $\begin{array}{c}\text { large } \\
C T\end{array}$ & $\frac{\text { all }}{C T}$ \\
\hline \hline FCFS & 579 & 0.8432 & 2.3294 & 1.9022 \\
\hline$w=0.8, K O=0$ & 386 & 0.4325 & 1.7532 & 1.3736 \\
\hline$w=1.0, K O=0$ & 449 & 0.4375 & 1.8540 & 1.4468 \\
\hline$w=0.8, K O=1$ & 6 & 0.3996 & 1.5715 & 1.2347 \\
\hline$w=1.0, K O=1$ & 5 & 0.3997 & 1.6219 & 1.2706 \\
\hline
\end{tabular}

For each scheduling, Table 1 lists the number of timeouts faced by small flows, along with the mean completion time (indicated by $\overline{C T}$ ) for small, large and all flows. We see that the number of timeouts encountered by small flows is highest for FCFS, followed by the schedulers without Knockout. This happens as some of the flows in $Q_{1}$, after being served with priority for the first $\theta$ packets, come back with more packets (due to a larger cwnd) and join $Q_{2}$, thereby increasing the total buffer occupancy. Without space priority, the packets of small flows are dropped when the buffer is full. With the Knockout policy, the timeouts are brought down tremendously as the packets of small flows are the last to experience drops. Fig. 4(b), which plots the worst-case completion time per flow size for small flows, also supports the necessity of giving space priority in addition to time priority (the figure does not plot the scenario of $\{w=0.8, K O=0\}$ for better clarity).

Comparing the mean CTs, it can be noted that the Knockout policy gives better results for all the means, compared to those without Knockout policy. Note that the prioritized service enjoyed by the first $\theta$ packets of a large flow helps in having a 'quicker' slow-start phase when compared to the FCFS-Droptail system. Similarly, non-strict schedulers give better performance (in terms of means) for large flows, compared to the strict counterparts (both with $K O=0$, and $K O=1)$. At the same time, the mean $\mathrm{CT}$ of small flows remain almost the same. With these comparisons, it becomes clear that a non-strict scheduler with Knockout buffer policy performs better than strict scheduler (strict $P S+P S$ ) without Knockout buffer. In general, these results also confirm a well-known result — SB scheduling outperforms FCFS scheduling in improving the delay performance.

\section{Performance Analysis of the Scheduler with Sampling}

This section analyzes the performance of the flow scheduler architecture, which combines the SB scheduler, the threshold-based sampling strategy and the Knockout buffer policy. For the scheduler, we set the weight $w$ to 0.8 . The results are compared to the SIFT scheme [1]. Note that SIFT does not use the Knockout policy; nor does it use a threshold to classify large flows. Instead, a sampled flow is considered 'large' and sent to $Q_{2}$; all other undetected flows go to $Q_{1}$. To see the degradation due to sampling, we also compare these schemes with the basic 
SB scheduling scheme (with no sampling). The packet-sampling probability is set to $1 / 100$ in the sampling schemes of both SIFT and our flow scheduler architecture. Here, we analyze the system under two traffic scenarios which differ in flow size distribution. Scenario 1 corresponds to the one considered before, where flow sizes were taken from a Pareto distribution. In Scenario 2, 85\% of flows are generated using an Exponential distribution with a mean $20 \mathrm{~KB}$; the remaining $15 \%$ are contributed by large flows using Pareto distribution with shape $\alpha=1.1$, and mean flow size set to $1 \mathrm{MB}$.

In the figures below, the name 'SB-SH' represents our flow scheduler architecture, coming from Size-Based scheduling using 'Sample and Hold'.

\subsection{Results with Traffic Scenario 1}

Figures 5(a), 5(b) 6(a) and 6(b) show the results. The conditional mean completion time curves for small flows in Fig. 5(a) reveal that sampling-cum-scheduling strategies (including SIFT) give improved performance for small flows in comparison to FCFS scheduling. This is anticipated, as most small flows go undetected and get prioritized. Even the maximum delay experienced by small flows using

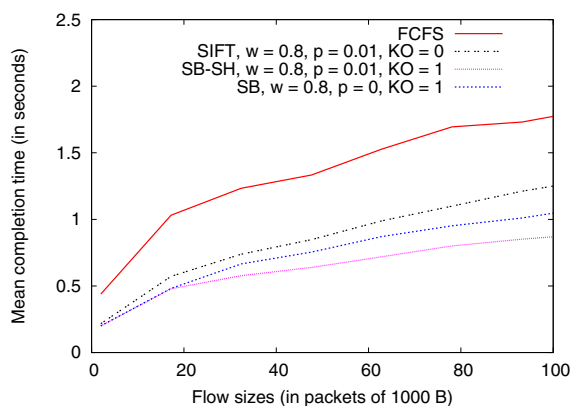

(a) small flows

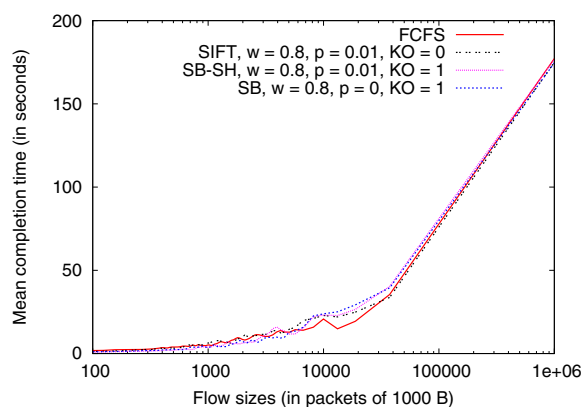

(b) large flows

Fig. 5. Conditional mean completion time

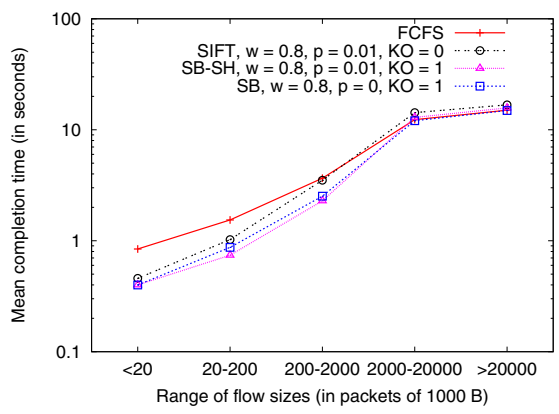

(a) Mean completion time

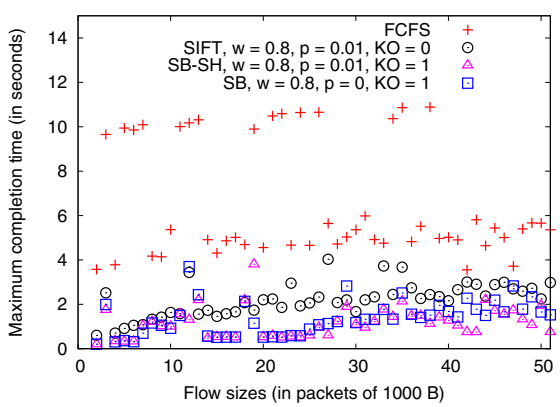

(b) Maximum completion time

Fig. 6. Other metrics 
Table 2. Comparison of different metrics

\begin{tabular}{|c||c|c|c|c|}
\hline Metrics & Small & small & large & all \\
& TOs & $\overline{C T}$ & $\frac{C T}{C T}$ \\
\hline \hline FCFS & 579 & 0.8432 & 2.3294 & 1.9022 \\
\hline$p=0.01, K O=0$, SIFT & 502 & 0.4585 & 1.9483 & 1.5200 \\
\hline$p=0.01, K O=1$, SB-SH & 5 & 0.3998 & 1.4406 & 1.1414 \\
\hline$p=0.0, K O=1$, SB & 6 & 0.3996 & 1.5715 & 1.2347 \\
\hline
\end{tabular}

sampling-cum-scheduling is lesser as seen in Fig. 6(b) In the figure, the completion time in SIFT is sometimes close to that of FCFS. These are cases when SIFT samples small flows and de-prioritizes them. Between the sampling-cumscheduling strategies, SB-SH scheme is seen to give smaller delay to small flows than SIFT, both in the mean case and in the worst case.

Additional metrics are compared in the Table 2, In all SB schedulers, the weights are the same $(w=0.8)$, and hence not made explicit in the table. The SIFT scheme induces large number of timeouts for small flows, as it gives no space priority to packets of small flows. In addition, a small flow that is sampled, gets de-prioritized in SIFT, leaving it to compete with the large flows. This is also clear from Fig. 6(b), which plots the maximum delay per size for small flows. From Fig. 6(a) and Table 2, it is seen that the delay for large flows is higher in SIFT than in the SB-SH scheme. Observe that we have the same sampling probability for both schemes. This means, a flow once sampled is de-prioritized immediately in SIFT; whereas a sampled flow still enjoys priority (both in time and space) for the next $\theta$ packets in SB-SH scheme. This helps the large flows to attain a large TCP cwnd faster (than in FCFS and SIFT).

Comparison of SB-SH scheme with the naive SB scheduling (without sampling), which shows that the former performs better than the latter, might appear to be surprising. But in fact, it is not - recall, that we have not tried to find the optimal threshold, $\theta$, in our study here. The false negatives that results from the sampling strategy increase the mean number of packets being served at $Q_{1}$, which is similar to increasing the threshold $\theta$. Increasing the threshold, increases the rate at which TCP $c$ wnd increases (due to negligible queueing delay and very few losses). To confirm, we performed SB scheduling (with Knockout policy, and without sampling) where $\theta$ was set to 100 packets. It was found that number of timeouts for small flows was 5 , and the mean CTs for small, large and all flows were 0.3997, 1.3740 and 1.0939 respectively. Except for the mean $\mathrm{CT}$ for small flow, which is almost the same for all, these values are better than all the results shown in Table 2 ,

\subsection{Results with Traffic Scenario 2}

Similar graphs were obtained for the second traffic scenario. We show only two plots here - figures $7(\mathrm{a})$ and $7(\mathrm{~b})$ and refer to an internal report for other figures [14. Table 3 compares other interested metrics. Comparing the values in 


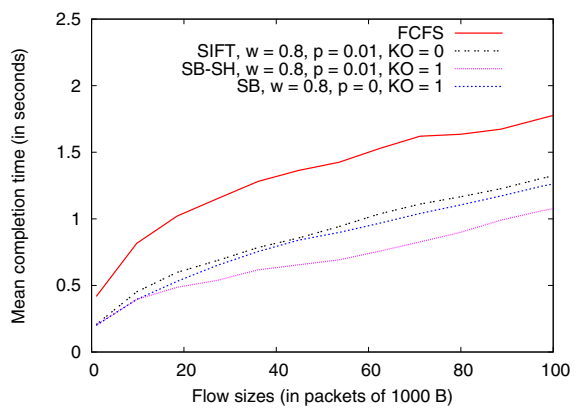

(a) Conditional mean completion time

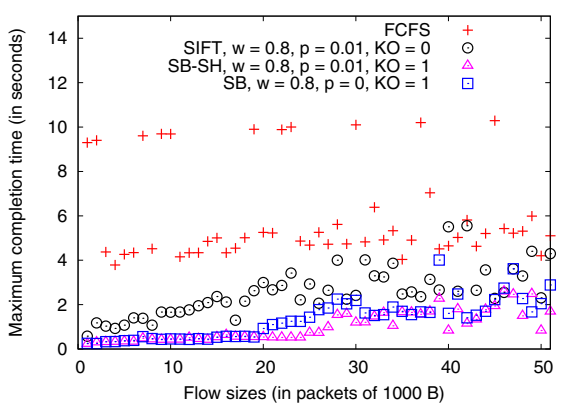

(b) Maximum completion time

Fig. 7. Metrics for small flows

Table 3. Comparison of different metrics

\begin{tabular}{|l||c|c|c|c|}
\hline Metrics & Small & small & large & all \\
& TOs & $\overline{C T}$ & $\overline{C T}$ & $\frac{C T}{C T}$ \\
\hline \hline FCFS & 792 & 0.7603 & 1.7491 & 1.2168 \\
\hline$p=0.01, K O=0$, SIFT & 778 & 0.4204 & 1.3195 & 0.8354 \\
\hline$p=0.01, K O=1$, SB-SH & 0 & 0.3671 & 1.2327 & 0.7666 \\
\hline$p=0.0, K O=1$, SB & 14 & 0.3698 & 1.3101 & 0.8039 \\
\hline
\end{tabular}

the table reveals that the results are similar to that with the first traffic scenario. Note that, as the number of small flows is higher in this scenario, SIFT gives a worse performance for the maximum completion time of small flows in this scenario (Fig. 7(b) in comparison to the previous scenario (Fig. 6(b)].

\section{Conclusions}

In this paper, we proposed a new flow scheduler architecture to improve the performance of flows in the Internet. Through arguments and simulations we have emphasized the importance of each of the modules in the architecture. The architecture is shown to improve the performance of flows in comparison to the naive FCFS scheduler. Besides, in comparison to SIFT, the flow scheduler architecture brings in better performance in terms of conditional mean completion time and timeouts for small flows, and mean CTs (for small, large, and all flows). Apart from these, the worst-case delay performance is also appealing.

In general, our study confirms previous observation that size-based scheduling induces negligible degradation to large flows. While sampling is known to be a practical solution in tracking large flows, here we also see that it does not affect the performance of small flows. This work opens different directions for future work. The parameters such as threshold $\theta$, weight $w$ and sampling probability $p$ were kept constant here. Finding the right values for each of these so as to obtain the optimal delay performance is dependent on the other two parameters. 
All of them have an influence on the mean queue length. A larger value of $\theta$ will result in a larger number of packets sent to $Q_{1}$, a smaller value of $w$ indicates a reduction in the service rate at $Q_{1}$, and decreasing the sampling probability will also increase the average number of packets of large flows served at $Q_{1}$. So, the variation in the average queue length (for a given load) can be used to decide the optimal values for these parameters.

\section{References}

1. Bonald, T., Oueslati-Boulahia, S., Roberts, J.: IP traffic and QoS control: the need for a flow-aware architecture. In: World Telecommunications Congress (September 2002)

2. Schrage, L.: A proof of the optimality of the Shortest Remaining Processing Time Discipline. Operations Research (16), 687-690 (1968)

3. Rai, I.A., Urvoy-Keller, G., Vernon, M.K., Biersack, E.W.: Performance analysis of las-based scheduling disciplines in a packet switched network. SIGMETRICS Perform. Eval. Rev. 32(1), 106-117 (2004)

4. Kleinrock, L.: Queueing Systems. Computer Applications, vol. II. Wiley Interscience, Hoboken (1976)

5. Guo, L., Matta, L.I.: The war between mice and elephants. In: ICNP 2001, pp. 180-188 (November 2001)

6. Rai, I.A., Biersack, E.W., Urvoy-Keller, G.: Size-based scheduling to improve the performance of short TCP flows. IEEE Network 19(1), 12-17 (2005)

7. Avrachenkov, K., Ayesta, U., Brown, P., Nyberg, E.: Differentiation Between Short and Long TCP Flows: Predictability of the Response Time. In: INFOCOM (2004)

8. Aalto, S., Ayesta, U., Nyberg-Oksanen, E.: Two-level processor-sharing scheduling disciplines: mean delay analysis. SIGMETRICS Perform. Eval. Rev. 32(1), 97-105 (2004)

9. Aalto, S., Ayesta, U.: Mean delay analysis of multi level processor sharing disciplines. In: INFOCOM (2006)

10. Zseby, T., et al.: RFC 5475: Techniques for IP Packet Selection (March 2009), http://www.rfc-editor.org/rfc/rfc5475.txt (Network Working Group)

11. Psounis, K., Ghosh, A., Prabhakar, B., Wang, G.: SIFT: A simple algorithm for tracking elephant flows, and taking advantage of power laws. In: 43rd Annual Allerton Conference on Control, Communication and Computing (2005)

12. Estan, C., Varghese, G.: New directions in traffic measurement and accounting. SIGCOMM Comput. Commun. Rev. 32(4), 323-336 (2002)

13. Chang, C.G., Tan, H.H.: Queueing analysis of explicit policy assignment pushout buffer sharing schemes for atm networks. In: Proceedings of the 13th IEEE Networking for Global Communications, vol. 2, pp. 500-509 (June 1994)

14. Divakaran, D.M., Carofiglio, G., Altman, E., Primet, P.V.B.: A flow scheduler architecture. Research Report 7133, INRIA (December 2009) 